\title{
ON THE DUAL OF A CERTAIN OPERATOR IDEAL
}

\author{
GERHARD RACHER
}

\begin{abstract}
For complex Banach spaces $E$ and $F$ and a real number $1<p<\infty$ let $S^{P}(E, F)$ denote the operator ideal obtained by complex interpolation between the nuclear and the compact operators. If $E$ and $F$ are reflexive and one of them has the approximation property the dual of $S^{p}(E, F)$ is shown to be $S^{p^{\prime}}\left(E^{\prime}, F^{\prime}\right), p^{\prime}$ conjugate to $p$.
\end{abstract}

Let $N$ denote the ideal of nuclear and $K$ the ideal of compact linear operators. By complex interpolation between $N$ and $K$ there is associated to every real number $1 \leqslant p \leqslant \infty$ the operator ideal $S^{p}$ in [4], i.e., $S^{p}(E, F)=$ $[N(E, F), K(E, F)]_{1 / p^{\prime}}$ for any complex Banach spaces $E$ and $F$ (that this definition gives the same as that of $[4$, p. 101] follows from [1, 9.3]). It is shown there that for any complex separable Hilbert space $H, S^{p}(H, H)$ consists of those compact operators whose moduli have $p$ th power summable eigenvalues. It is then a classical result of Schatten and von Neumann that the Banach space dual of $S^{p}(H, H)$ may be identified with $S^{p^{\prime}}(H, H)$. Here we are showing

Proposition. Let $E$ and $F$ be complex Banach spaces and $p$ a real number with $1<p<\infty, 1 / p^{\prime}+1 / p=1$. If $E$ and $F$ are reflexive and one of them has the approximation property then the dual of $S^{p}(E, F)$ may be identified isometrically with $S^{p^{\prime}}\left(E^{\prime}, F^{\prime}\right)$, the pairing given by $\left(S \in S^{p^{\prime}}\left(E^{\prime}, F^{\prime}\right)\right.$ and $\left.T \in S^{p}(E, F)\right):\langle T, S\rangle=$ $\operatorname{trace}\left(T^{\prime} \circ S\right)$ when $E$ has the approximation property; $\langle T, S\rangle=\operatorname{trace}\left(S \circ T^{\prime}\right)$ when $F$ has it.

In particular the space $S^{p}(E, F)$ is reflexive.

Proof. We shall use the notation of [1] without further explanation. Let us assume that $E$ has the approximation property.

First step. Since $E$ is reflexive $E^{\prime}$ has it too by [3 Proposition 36.1]. By Satz 3 and Satz 7 in [4], for every $S \in S^{p^{\prime}}\left(E^{\prime}, F^{\prime}\right)$ and $T \in S^{p}(E, F)$ the product $T^{\prime} \circ S$ is nuclear, and $\left\|T^{\prime} \circ S\right\|_{N} \leqslant\left\|T^{\prime}\right\|_{S^{p}}\|S\|_{S^{\prime}} \leqslant\|T\|_{S^{p}}\|S\|_{S^{\prime \prime}}$ Since $E^{\prime}$ has the approximation property, $\operatorname{trace}\left(T^{\prime} \circ S\right)$ is well defined and $<\left\|T^{\prime} \circ S\right\|_{N}<$ $\|T\|_{S^{p}}\|S\|_{S^{p}}$ such that $\beta: \quad S^{p^{\prime}}\left(E^{\prime}, F^{\prime}\right) \rightarrow S^{p}(E, F)^{\prime}$, given by $\langle T, \beta S\rangle=$ $\operatorname{trace}\left(T^{\prime} \circ S\right)$, is a linear contraction.

Second step. $\beta$ is an isometry. Since the linear mappings of finite rank are dense in $S^{p^{\prime}}\left(E^{\prime}, F^{\prime}\right)$ it suffices to show that

$$
\|S\|_{S^{p^{\prime}\left(E^{\prime}, F^{\prime}\right)}}<\|\beta S\|_{S^{p}(E, F)^{\prime}}
$$

Received by the editors January 22, 1980.

AMS (MOS) subject classifications (1970). Primary 46L20; Secondary 46E35.

Key words and phrases. Reflexivity, operator ideals, nuclear and compact operators, complex interpolation. 
for any such map. So let $S: E^{\prime} \rightarrow F^{\prime}$ be a linear map of finite rank. By the theorem of Hahn-Banach there exists a linear form $L$ in $S^{p^{\prime}}\left(E^{\prime}, F^{\prime}\right)^{\prime}$ of norm 1 with $\|S\|_{S^{p^{\prime}}}=\langle S, L\rangle=\operatorname{trace}\left(L^{\prime} \circ S\right)$, when we identify $L$ with the corresponding bounded linear map from $E$ to $F$. By definition and the duality theorem 12.1 in [1] one has

$$
S^{p^{\prime}}\left(E^{\prime}, F^{\prime}\right)^{\prime}=\left[N\left(E^{\prime}, F^{\prime}\right)^{\prime}, K\left(E^{\prime}, F^{\prime}\right)^{\prime}\right]^{1 / p}
$$

since $N\left(E^{\prime}, F^{\prime}\right)$ is dense in $K\left(E^{\prime}, F^{\prime}\right)$ because of the approximation property of $E^{\prime \prime}=E$. Identifying $N\left(E^{\prime}, F^{\prime}\right)^{\prime}=\left(E^{\prime \prime} \hat{\otimes} F^{\prime}\right)^{\prime}=\left(E \hat{\otimes} F^{\prime}\right)^{\prime}=H(E, F)$, the space of bounded linear mappings from $E$ to $F$, and $K\left(E^{\prime}, F^{\prime}\right)^{\prime}=\left(E^{\prime \prime} \check{\otimes} F^{\prime}\right)^{\prime}=$ $\left(E \otimes F^{\prime}\right)^{\prime}=I(E, F)$, the space of integral mappings from $E$ to $F$, which coincides with $N(E, F)$, since $F$ is reflexive [3, Théorème 10.1], we obtain

$$
S^{p^{\prime}}\left(E^{\prime}, F^{\prime}\right)^{\prime}=[N(E, F), H(E, F)]^{t} \quad \text { with } t=1-1 / p=1 / p^{\prime} .
$$

So for every $\varepsilon>0$ we can find a function $h$ in $\overline{\mathscr{F}}(N(E, F), H(E, F))$ with $\|h\|_{\overline{\mathscr{F}}}<1$ $+\varepsilon$ and whose derivative $h^{\prime}(t)$ at the point $t$ equals $L$.

For this function $h$ we construct in the usual manner (cf. [2]) a sequence of functions $h_{n}$ in $\mathscr{F}(N(E, F), H(E, F))$ with $\lim _{n \rightarrow \infty} h_{n}(t)=h^{\prime}(t)$; for example,

$$
h_{n}(z)=\exp \left(z^{2} / n\right)[h(z+i / n)-h(z)] n / i \text { for } 0<\operatorname{Re} z<1, n>1 \text {. }
$$

$\left\|h_{n}\right\|_{\mathcal{F}} \leqslant e^{1 / n}\|h\|_{\overline{\mathcal{F}}}$ for every $n$. By 9.3 in [1] (second line from below) we have $\mathscr{F}(N(E, F), \quad H(E, F))=\mathscr{F}(N(E, F), K(E, F))$ isometrically so that $h_{n}(t) \in$ $[N(E, F), K(E, F)]_{t}=S^{p}(E, F)$ and $\left\|h_{n}(t)\right\|_{S^{p}}<\left\|h_{n}\right\|_{\mathscr{F}}<e^{1 / n}(1+\varepsilon)$ for every $n$. All together one has

$$
\begin{aligned}
\|S\|_{S^{p^{\prime}\left(E^{\prime}, F^{\prime}\right)}} & =\operatorname{trace}\left(L^{\prime} \circ S\right)=\operatorname{trace}\left(\left[h^{\prime}(t)\right]^{\prime} \circ S\right) \\
& =\lim \operatorname{trace}\left([(h(t+i / n)-h(t)) n / i]^{\prime} \circ S\right) \\
& =\lim \operatorname{trace}\left(\exp \left(-t^{2} / n\right)\left[h_{n}(t)\right]^{\prime} \circ S\right) \\
& =\lim \exp \left(-t^{2} / n\right)\left\langle h_{n}(t), \beta S\right\rangle \\
& \leqslant \lim \sup \exp \left(-t^{2} / n\right)\left\|h_{n}(t)\right\|_{S^{p}}\|\beta S\|_{S^{p}(E, F)^{\prime}} \\
& \leqslant \lim \sup \exp \left(\left(1-t^{2}\right) / n\right)(1+\varepsilon)\|\beta S\|_{S^{p}(E, F)^{\prime}} \\
& \leqslant(1+\varepsilon)\|\beta S\|_{S^{p}(E, F)^{\prime}}
\end{aligned}
$$

i.e.,

$$
\|S\|_{S^{\rho^{\prime}\left(E^{\prime}, F^{\prime}\right)}}<\|\beta S\|_{S^{p}(E, F)^{\prime}}
$$

Since this inequality obtains for every $S \in S^{p^{\prime}}\left(E^{\prime}, F^{\prime}\right)$, the isometry of $\beta$ follows in conjunction with the contractivity of $\beta$.

Third step. The image of $\beta$ is dense in $S^{p}(E, F)^{\prime}$. First of all note that $S^{p}(E, F)^{\prime}$ is equal to $\left[N(E, F)^{\prime}, K(E, F)^{\prime}\right]^{1 / p^{\prime}}=\left[N\left(E^{\prime}, F^{\prime}\right), H\left(E^{\prime}, F^{\prime}\right)\right]^{1 / p^{\prime}}$ by the duality theorem (cf. Second step). Now by the last formula in 9.3 of [1] this last space is equal to $\left[N\left(E^{\prime}, F^{\prime}\right), K\left(E^{\prime}, F^{\prime}\right)\right]^{1 / p^{\prime}}$ which by definition is contained in $N\left(E^{\prime}, F^{\prime}\right)+$ $K\left(E^{\prime}, F^{\prime}\right)=K\left(E^{\prime}, F^{\prime}\right)$. Since $K\left(E^{\prime}, F^{\prime}\right)$ is the closure of the operators of finite rank and these are contained in $S^{p^{\prime}}\left(E^{\prime}, F^{\prime}\right), \beta$ has dense image. 
So $\beta$ : $S^{p^{\prime}}\left(E^{\prime}, F^{\prime}\right) \rightarrow S^{p}\left(E, F^{\prime}\right)$ is an isometric isomorphism. Since the proof works as well when $F$ (hence $F^{\prime}$ ) has the approximation property the proposition is established.

Let now $G$ denote a compact group with normalized Haar measure and $L^{q}(G)$ its complex Lebesgue spaces. Let $S_{G}^{p}\left(L^{q}(G), L^{q}(G)\right)$ denote the set of those operators in $S^{p}\left(L^{q}(G), L^{q}(G)\right)$ which are (left-or-right-) translation invariant under $G$. Since it is a closed subspace of $S^{p}\left(L^{q}(G), L^{q}(G)\right)$ one arrives at

Corollary. Let $G$ be a compact group and $1<p, q<\infty$. Then the two-sided $q$-Segal algebras $S_{G}^{p}\left(L^{q}(G), L^{q}(G)\right)$ are reflexive Banach spaces.

The assertion of the corollary remains true for $q=1$, since then

$$
S_{G}^{p}\left(L^{1}(G), L^{1}(G)\right)=L^{p^{\prime}}(G)
$$

which was the starting point for this note (cf. [5]).

Acknowledgement. I am much indebted to Dr. Michael Cwikel from Technion Haifa for making me familiar with the technique used in Second step and for kindly sending me a proof of the last formula of 9.3 in [1], communicated privately to him by A. P. Calderón. The proof is reproduced in J. Bergh, On the relation between the two complex methods of interpolation, to appear in Indiana Univ. Math. J.

\section{REFERENCES}

1. A. P. Calderón, Intermediate spaces and interpolation, the complex method, Studia Math. 24 (1964), 113-190.

2. M. Cwikel, Complex interpolation spaces, a discrete definition and reiteration, Indiana Univ. Math. J. 27 (1978), 1005-1009.

3. A. Grothendieck, Produits tensoriels topologiques et espaces nucléaires, Mem. Amer. Math. Soc., no 16, 1965.

4. A. Pietsch und H. Triebel, Interpolationstheorie für Banachideale von beschränkten linearen Operatoren, Studia Math. 31 (1968), 95-109.

5. G. Racher, Beispiele von Segalalgebren auf kompakten Gruppen, Universität Salzburg, 1978.

Petersbrunnerstrasse 19, A-5020 Salzburg, Austria 